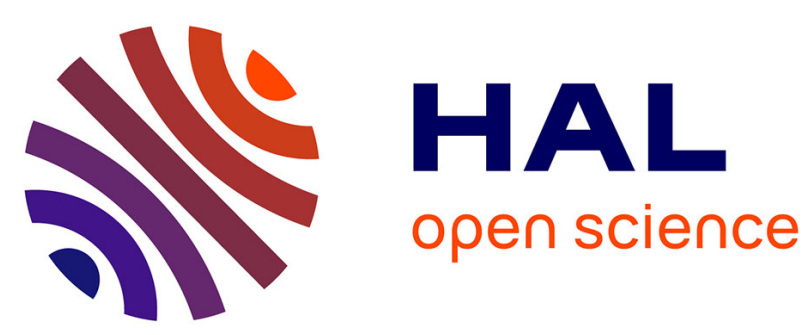

\title{
Existence of Pulses for Local and Nonlocal Reaction-Diffusion Equations
}

Nathalie Eymard, Vitaly Volpert, Vitali Vougalter

\section{To cite this version:}

Nathalie Eymard, Vitaly Volpert, Vitali Vougalter. Existence of Pulses for Local and Nonlocal Reaction-Diffusion Equations. Journal of Dynamics and Differential Equations, 2015, 10.1007/s10884015-9487-1 . hal-01237744

\section{HAL Id: hal-01237744 \\ https://hal.science/hal-01237744}

Submitted on 3 Dec 2015

HAL is a multi-disciplinary open access archive for the deposit and dissemination of scientific research documents, whether they are published or not. The documents may come from teaching and research institutions in France or abroad, or from public or private research centers.
L'archive ouverte pluridisciplinaire HAL, est destinée au dépôt et à la diffusion de documents scientifiques de niveau recherche, publiés ou non, émanant des établissements d'enseignement et de recherche français ou étrangers, des laboratoires publics ou privés. 


\title{
Existence of pulses for local and nonlocal reaction-diffusion equations
}

\author{
Nathalie Eymard ${ }^{1}$, Vitaly Volpert ${ }^{1}$, Vitali Vougalter $^{2}$ \\ ${ }^{1}$ Institut Camille Jordan, UMR 5208 CNRS, University Lyon 1 \\ 69622 Villeurbanne, France \\ E-mail addresses: eymard@math.univ-lyon1.fr, volpert@math.univ-lyon1.fr \\ ${ }^{2}$ Department of Mathematics, University of Toronto \\ Toronto, Ontario, M5S 2E4 Canada \\ E-mail address: vitali@math.toronto.edu
}

\begin{abstract}
Reaction-diffusion equations with a space dependent nonlinearity are considered on the whole axis. Existence of pulses, stationary solutions which vanish at infinity, is studied by the Leray-Schauder method. It is based on the topological degree for Fredholm and proper operators with the zero index in some special weighted spaces and on a priori estimates of solutions in these spaces. Existence of solutions is related to the speed of travelling wave solutions for the corresponding autonomous equations with the limiting nonlinearity.
\end{abstract}

Key words: reaction-diffusion equation, existence of pulse solutions, Leray-Schauder method AMS subject classification: 35K57, 35A16, 92D15

\section{Introduction}

Existence of solutions of the semilinear elliptic equation

$$
\Delta w+F(w, x)=0
$$

in $\mathbb{R}^{n}, n \geq 1$ depends on the nonlinearity $F(w, x)$. It is studied in detail for polynomial functions $F(w, x)=w^{p}$ where existence of solutions is determined by the values of $n$ and $p$ [4], [5], [6], [7], [8]. Another typical example is given by the nonlinearity $F(w, x)=$ $-a(x)|w|^{p-2} w+\lambda b(x)|w|^{q-2} w$, where $a(x)$ and $b(x)$ are positive functions and $\lambda$ is a positive parameter. Under some additional conditions it is proved that there exists a value of $\lambda$ for which a nontrivial solution of this equation exists in the corresponding function space. In some cases it can be proved that this solution is radial $(n>1)$ and non-negative. The review of methods and results can be found in [1], [9]. 
In the second example considered above, the nonlinearity has variable sign and the trivial solution $w=0$ can be stable. In this case, nontrivial solutions, if they exist, do not bifurcate from the trivial one. We can expect that they separate basins of attraction of the trivial solution and of some other solution or infinity (blow up solution). These properties can be clearly seen in the autonomous case, for example $F(w, x)=-a w+b w^{2}$, where $a$ and $b$ are some positive constants. In this case, equation (1.1) has a positive solution (pulse) which vanish at infinity [2]. It is unstable as a stationary solution of the corresponding parabolic equation. Solution of the Cauchy problem with a perturbed initial condition will converge either to 0 or grow to infinity. Moreover, such pulse solutions exist for any values of parameters $a$ and $b$ and not only for some values of parameters as in the example above. In the one-dimensional case, they can be easily found analytically.

In this work we will study existence of pulse solutions for a nonautonomous nonlinearity. We will consider the one-dimensional equation on the whole axis

$$
w^{\prime \prime}+F(w, x)=0
$$

and we will look for its classical solutions with the limits

$$
w( \pm \infty)=0 .
$$

Similar to the example above, we will see that such solutions can separate basin of attraction of the trivial solution and of some other solution. In fact, existence of solutions of problem (1.2), (1.3) will be determined by the properties of travelling waves for the corresponding autonomous equation with the limiting function $F_{0}(w)=\lim _{|x| \rightarrow \infty} F(w, x)$. The typical form of the function $F$ considered in this work is given by the example from population dynamics

$$
F(w, x)=w^{2}(1-w)-\sigma(x) w,
$$

where the first term in the right-hand side describes the reproduction of the population, the second term its mortality. The mortality rate $\sigma(x)$ is a positive function which depends on the space variable. We can consider the nonlinearity in a more general form. The conditions on it will be specified below.

We will use function (1.4) in order to explain the main features of this problem. Consider first the case where $\sigma(x) \equiv \sigma_{0}$ is a constant. Set

$$
F_{0}(w)=w^{2}(1-w)-\sigma_{0} w .
$$

Suppose that $0<\sigma_{0}<1 / 4$ and consider the zeros of this function, $w_{0}=0, w_{1}$ and $w_{2}$ are solutions of the equation $w(1-w)=\sigma_{0}, 0<w_{1}<w_{2}$. If

$$
\int_{0}^{w_{2}} F_{0}(w) d w>0
$$

then the equation

$$
w^{\prime \prime}+F_{0}(w)=0
$$


with the limits $w( \pm \infty)=0$ has two solutions. The trivial solution $W_{0}(x) \equiv 0$ and a positive solution $W_{1}(x)$ which can be easily found analytically. Its explicit form is not essential for us. In the case of the inequality

$$
\int_{0}^{w_{2}} F_{0}(w) d w<0
$$

there exists only trivial solution. Finally, if

$$
\int_{0}^{w_{2}} F_{0}(w) d w=0
$$

then equation (1.6) does not have nontrivial solutions, which vanish at infinity, but there is a solution with the limits

$$
w(-\infty)=w_{2}, \quad w(+\infty)=0 .
$$

Let us note these three cases are related to the speed of travelling waves, that is solutions of the equation

$$
w^{\prime \prime}+c w^{\prime}+F_{0}(w)=0
$$

with limits (1.9). Here $c$ is the wave speed. Its sign can be determined multiplying the equation by $w^{\prime}$ and integrating from $-\infty$ to $+\infty$. If condition (1.5) is satisfied, then $c>0$. If the inequality is opposite, then $c<0$. Finally, in the case (1.8), $c=0$. We will see that properties of solutions of problem (1.2), (1.3) depend on the sign of the wave speed.

Condition (1.5). In this case, the question about the existence of solution can be formulated in the following way. If we change the function $\sigma(x)$ by a continuous deformation starting from the constant $\sigma_{0}$, will the nontrivial solution persist? We will study this question by a Leray-Schauder method which is based on topological degree and a priori estimates of solutions. Topological degree in unbounded domains is defined in weighted spaces, in the space without weight it may not exist. Therefore the estimates should also be obtained in the weighted spaces. Usual estimates in Hölder or Sobolev spaces are not sufficient.

Let us explain in more detail what estimates we need in order to prove the existence of solutions and how they can be obtained. Consider equation (1.2) with the function $\sigma_{\tau}(x)$ which depends on a parameter $\tau$. Suppose that there exists a limit $\sigma_{0}=\lim _{x \rightarrow \pm \infty} \sigma_{\tau}(x)$. Let $E=C_{\mu}^{2+\alpha}(\mathbb{R})$ be the weighted Hölder space with the norm $\|u\|_{E}=\|u \mu\|_{C^{2+\alpha}(\mathbb{R})}$, where $\mu(x)=\sqrt{1+x^{2}}$. If the usual Hölder norm $\|u\|_{E_{0}}, E_{0}=C^{2+\alpha}(\mathbb{R})$ is bounded and the solution decays exponentially at infinity, then the weighted Hölder norm is also bounded. The $E_{0^{-}}$ norm of the solution can be estimated by the usual methods, exponential decay of solution at infinity follows from the assumption $F_{0}^{\prime}(0)<0$. However this is not sufficient for a uniform estimate in the weighted Hölder space. Consider a family of solutions $u_{\tau}$ which depends on a parameter $\tau$. If the weighted norm $\|u\|_{E}$ tends to infinity as $\tau \rightarrow \tau_{0}$, then it can be verified that equation (1.6) has a nonzero solution, and $u_{\tau}$ converges to this solution. Under 
some additional conditions, we will prove that this convergence cannot occur and will obtain, by this, a priori estimates of solutions. We will use here monotonicity of solutions on the half-axis.

Condition (1.7). The situation is different in the case of condition (1.7). Equation (1.6) does not have nontrivial solutions vanishing at infinity. A nontrivial solution can bifurcate from the trivial solution. Condition (1.7) will allow us to obtain a priori estimates of nontrivial solutions in the weighted norm. Hence if the trivial solution becomes unstable, then we will obtain the existence of a nontrivial solution. Let us note that for function (1.4) the trivial solution becomes unstable if the function $\sigma(x)$ is negative for some $x$. This does not correspond to the biological meaning of this example. We consider this case in view of other possible applications and more general functions $F$.

Thus, equality (1.8) separates two cases where we prove existence of solutions. In the case where this equality is satisfied, the method to prove existence of solutions developed in this work is not applicable.

Nonlocal equations. Besides equation (1.2) we will also study the nonocal equation

$$
w^{\prime \prime}+F(w, x, I)=0, \quad I(w)=\int_{-\infty}^{\infty} w(x) d x
$$

with the typical example of nonlinearity

$$
F(w, x, I)=w^{2}(1-I)-\sigma(x) w .
$$

This case is quite similar to the previous one, and analysis of the existence of solutions is based on the previous results. However, due to the integral term, the number of solutions can change, and some of them can become stable [12]. Though we do not study stability of solutions in this work, this is an important argument for the investigation of pulse solutions. Existence and dynamics of pulses for autonomous nonlocal reaction-diffusion equations are studied in [13], [15] (see also [16]).

Leray-Schauder method. Existence of solutions will be proved by the Leray-Schauder method based on the topological degree for elliptic problems in unbounded domains. Let us recall that the Leray-Schauder degree is not generally applicable in this case. It can be used under some special conditions on the coefficients which allow the reduction of the corresponding operator to the compact operator [9]. In our case this approach is not applicable. We will use the degree construction for Fredholm and proper operators with the zero index [11]. This construction requires the introduction of special weighted spaces. Therefore a priori estimates of solutions required for the Leray-Schauder method should also be obtained in the weighted spaces. This is a special type of estimates where the boundedness of the solution and of its derivatives is not sufficient. These estimates will be obtained for monotone solutions and will require separation of monotone and non-monotone solutions (explained 
below). This approach is inspired by the method of proof of travelling waves for monotone and locally monotone systems [10].

In the next section we will introduce function spaces and operators, we will obtain a priori estimates of solutions and prove the main existence result (Theorem 2.7). It corresponds to the case (1.5) in the example considered above. In Section 3 we will discuss another possible case which correspond to inequality (1.7). Nonlocal equations will be considered in Section 4.

\section{Monotone solutions on the half-axis}

We begin with the problem on the half-axis. We consider equation (1.2) for $x>0$ with the Neumann boundary condition and look for a solution decaying at infinity:

$$
w^{\prime}(0)=0, \quad w(+\infty)=0 .
$$

This problem can be extended to the whole axis by symmetry. We will assume that

$$
F(0, x)=0, \quad x \geq 0 ; \quad F_{x}^{\prime}(w, x)<0, \quad w>0, \quad x \geq 0
$$

and for some $\epsilon>0$

$$
F_{w}^{\prime}(0, x) \leq-\epsilon, \quad \forall x \geq 0 .
$$

Moreover, there exists $w_{+}>0$ such that

$$
F(w, x)<0, \quad \forall x \geq 0, w>w_{+} .
$$

\subsection{Operators and spaces}

We will use the Leray-Schauder method to prove the existence of solutions. We need to introduce the operators, function spaces, construct a continuous deformation of the operator and obtain a priori estimates of solutions. Operators and spaces should be defined in such a way that the topological degree exists for them. We suppose that the function $F(w, x)$ is sufficiently smooth with respect to both variables. We can assume for simplicity that it is infinitely differentiable with all derivatives bounded. Consider the operator

$$
A(w)=w^{\prime \prime}+F(w, x)
$$

acting from the space

$$
E_{1}=\left\{u \in C_{\mu}^{2+\alpha}\left(\mathbb{R}_{+}\right), \quad u^{\prime}(0)=0\right\}
$$

into the space $E_{2}=C_{\mu}^{\alpha}\left(\mathbb{R}_{+}\right)$. Here $C_{\mu}^{k+\alpha}\left(\mathbb{R}_{+}\right)$is a weighted Hölder space with the norm 


$$
\|u\|_{C_{\mu}^{k+\alpha}\left(\mathbb{R}_{+}\right)}=\|u \mu\|_{C^{k+\alpha}\left(\mathbb{R}_{+}\right)}
$$

where $\mu(x)$ is a weight function. We will set $\mu(x)=\sqrt{1+x^{2}}$. Construction of the topological degree for such spaces and operators can be found in [11].

\subsection{Separation of monotone solutions}

We will obtain a priori estimates of solutions for monotonically decreasing solutions. Therefore we need to separate them from non-monotone solutions. We understand this separation in the following sense. Consider a solution $w_{\tau}(x) \not \equiv 0$ which depends on parameter $\tau$. The dependence on $\tau$ is continuous in the norm $C^{1}\left(\mathbb{R}_{+}\right)$. Suppose that the solution $w_{\tau}(x)$ is monotonically decreasing for $\tau<\tau_{0}$ and it is not monotonically decreasing for $\tau>\tau_{0}$. We will prove that this assumption leads to a contradiction.

We proceed by contradiction. Then there is a sequence $\tau_{n} \rightarrow \tau_{0}$ and the sequence of solutions $w_{n}(x)=w_{\tau_{n}}(x)$ such that these functions are not monotone and the function $w_{0}(x)=w_{\tau_{0}}(x)$ is monotone. Therefore there exists a sequence $x_{n}$ such that $w_{n}^{\prime}\left(x_{n}\right)=0$. Without loss of generality, we can suppose that $x_{n} \rightarrow x_{0}$, where one of the following three cases takes place: $0<x_{0}<\infty, x_{0}=\infty, x_{0}=0$. We will show that all of them lead to contradiction.

Finite value of $x_{0}$. Consider first the case where $0<x_{0}<\infty$. Then $w_{0}^{\prime}\left(x_{0}\right)=0$ and $w_{0}^{\prime}(x) \leq 0$ for all $x \geq 0$. Set $u(x)=-w_{0}^{\prime}(x)$. Differentiating equation (1.2), we get

$$
u^{\prime \prime}+F_{w}^{\prime}\left(w_{0}, x\right) u-F_{x}^{\prime}\left(w_{0}, x\right)=0 .
$$

Since $u(x) \geq 0$ for all $x \geq 0, u\left(x_{0}\right)=0, F_{x}^{\prime}<0$, then we obtain a contradiction in signs in the last equation. If the inequality in $(2.2)$ is not strict, that is $F_{x}^{\prime} \leq 0$, then the last equation contradicts the maximum principle.

Remark. Condition (2.2) can be replaced by a weaker condition. Suppose that

$$
F(w, x)=0 \Rightarrow F_{x}^{\prime}(w, x)<0, \quad w>0, \quad x \geq 0 .
$$

Therefore the derivative $F_{x}^{\prime}$ is negative not everywhere as in condition (2.2) but only at zero lines of the function $F$. In this case, since $w_{0}^{\prime \prime}\left(x_{0}\right)=0$, then $F\left(w_{0}\left(x_{0}\right), x_{0}\right)=0$. By virtue of condition $(2.6)$, we have $F_{x}^{\prime}\left(w_{0}\left(x_{0}\right), x_{0}\right)<0$. Differentiating equation (1.2) and taking into account that $w_{0}^{\prime}\left(x_{0}\right)=0$, we obtain

$$
w_{0}^{\prime \prime \prime}\left(x_{0}\right)=-F_{x}^{\prime}\left(w_{0}\left(x_{0}\right), x_{0}\right)>0 .
$$

Hence $w_{0}^{\prime}(x)>0$ in some neighborhood of the point $x_{0}$. This contradicts the assumption that $w_{0}^{\prime}(x) \leq 0$. 
Infinite value of $x_{0}$. We consider now the case where $x_{n} \rightarrow \infty$. Since $w_{n}(x) \rightarrow w_{0}(x)$ in $C^{1}\left(\mathbb{R}_{+}\right)$and $w_{0}(x) \rightarrow 0$ as $x \rightarrow \infty$, then $w_{n}\left(x_{n}\right) \rightarrow 0$ as $n \rightarrow \infty$. If $w_{n}\left(x_{n}\right)>0$, then $F\left(w_{n}\left(x_{n}\right), x_{n}\right)<0$ and $w_{n}^{\prime \prime}\left(x_{n}\right)>0$. Hence any positive extremum of the functions $w_{n}(x)$ is a minimum. Therefore they cannot converge to zero at infinity. Similarly, if $w_{n}\left(x_{n}\right)<0$, then it is a maximum and, as before, the function cannot converge to zero at infinity. Finally, if $w_{n}\left(x_{n}\right)=0$, then by virtue of condition (2.2) we have that $F\left(w_{n}\left(x_{n}\right), x_{n}\right)=0, w_{n}^{\prime \prime}\left(x_{n}\right)=0$. Therefore $w_{n}(x) \equiv 0$. This contradicts our assumption that all solutions $w_{\tau}(x)$ are nontrivial.

Zero value of $x_{0}$. Let us consider the last case where $x_{n} \rightarrow x_{0}=0$. We note that $F\left(w_{0}(0), 0\right)>0$. Indeed, if $F\left(w_{0}(0), 0\right)<0$, then $w_{0}^{\prime \prime}(0)>0$. Since $w_{0}^{\prime}(0)=0$, then this function cannot be monotonically decreasing.

If $F\left(w_{0}(0), 0\right)=0$, then $w_{0}^{\prime \prime}(0)=0$. The function $u(x)=-w_{0}^{\prime}(x)$ satisfies equation (2.5) where $F_{x}^{\prime}<0$. Since $u(x) \geq 0$ for all $x \geq 0, u(0)=0$ and $u^{\prime}(0)=0$, then we obtain a contradiction with the Hopf lemma which affirms that $u^{\prime}(0)>0$. In the case of the generalized condition (2.6), we get inequality (2.7). It contradicts the assumption that the function $w_{0}(x)$ is decreasing.

Thus, we proved that $F\left(w_{0}(0), 0\right)>0$. Therefore the same inequality holds in some neighborhood of $x=0$. Since $w_{n}(x)$ converges uniformly to $w_{0}(x)$, then $w_{n}^{\prime \prime}(x)<0$ in the interval $0<x<\delta$ with some positive $\delta$ independent of $n$. This assertion contradicts the assumption that $w_{n}^{\prime}\left(x_{n}\right)=0$ and $x_{n} \rightarrow 0$ as $n \rightarrow \infty$. We proved the following theorem.

Theorem 2.1 (Separation of monotone solutions.) There exists a positive number $r$ such that for any solution of problem (2.8), (2.9), which depends on a parameter $\tau$, we have

$$
\left\|w_{M}-w_{N}\right\|_{E_{1}} \geq r
$$

for any monotone solution $w_{M}$ and any nonmonotone solution $w_{N}$ of this problem. This estimate does not depend on $\tau$.

\subsection{A priori estimates of monotone solutions}

We consider the equation

$$
w^{\prime \prime}+F_{\tau}(w, x)=0
$$

on the half-axis $x>0$ with the Neumann boundary condition:

$$
w^{\prime}(0)=0 .
$$

We will look for its solution decaying at infinity, $w(+\infty)=0$. In order to simplify the presentation, we suppose that the function $F_{\tau}(w, x)$ is infinitely differentiable with respect to all variables $w, x, \tau$,

$$
F_{\tau}(0, x)=0, \quad x \geq 0 ; \quad \frac{\partial F_{\tau}(w, x)}{\partial x}<0, \quad w>0, \quad x \geq 0
$$


and

$$
\frac{\partial F_{\tau}(0, x)}{\partial w} \leq-\epsilon, \quad x \geq 0, \quad \tau \in[0,1]
$$

for some $\epsilon>0$. Moreover, there exists $w_{+}>0$ such that

$$
F_{\tau}(w, x)<0, \quad \forall w>w_{+}, \quad x \geq 0, \quad \tau \in[0,1]
$$

Let $w_{\tau}(x)$ be positive solutions of problem (2.8), (2.9) decaying at infinity. From condition (2.12) it follows that $w_{\tau}(x) \leq w_{+}$for all $x$ and $\tau$. Indeed, since we consider montonically decreasing solutions, it is sufficient to verify that $w_{\tau}(0) \leq w_{+}$. If the opposite inequality holds, then, by virtue of the equation, $w_{\tau}^{\prime \prime}(0)>0$, and this function is not decreasing.

Since the function $F_{\tau}(w, x)$ is sufficiently smooth with respect to $w, x$ and $\tau$, then solutions are uniformly bounded in $C^{2}\left(\mathbb{R}_{+}\right)$and the dependence on $\tau$ is continuous in $C^{1}\left(\mathbb{R}_{+}\right)$. In order to prove that solutions are uniformly bounded in the weighted Hölder norm, it is sufficient to verify that the weighted norm

$$
\left\|w_{\tau}\right\|_{\mu}=\|w \mu\|_{C\left(\mathbb{R}_{+}\right)}
$$

of these solutions is uniformly bounded. Here $\mu(x)=\sqrt{1+x^{2}}$.

Let $\epsilon>0$ be sufficiently small such that $F_{\tau}(w, x)<0$ for $0<w<\epsilon$ and all $x \geq 0$. Such $\epsilon$ exists by virtue of condition (2.11). Since $w_{\tau}(x)$ is a decreasing function and, obviously, $w_{\tau}(0)>\epsilon$ (otherwise $F_{\tau}\left(w_{\tau}(0), 0\right)<0$ and $w_{\tau}^{\prime \prime}(0)>0$ ), then there exists a unique solution of the equation $w_{\tau}(x)=\epsilon$. Denote it by $x_{\tau}$.

The solutions $w_{\tau}(x)$ admit a uniform exponential estimate for $x>x_{\tau}$. Therefore if $x_{\tau}$ is uniformly bounded, then we obtain a uniform estimate of the norm $\left\|w_{\tau}\right\|_{\mu}$.

Suppose that the values $x_{\tau}$ are not uniformly bounded. Then there exists a sequence $\tau_{n}$ for which $x_{n}=x_{\tau_{n}} \rightarrow \infty$. Without loss of generality we can assume that $\tau_{n} \rightarrow \tau_{0}$ for some $\tau_{0} \in[0,1]$. We will consider the corresponding sequence of solutions $w_{n}(x)=w_{\tau_{n}}(x)$. It has a subsequence locally convergent to a solution $w_{0}(x)$ such that $w_{0}^{\prime}(0)=0$ and $w_{0}(\infty)>0$. The latter follows from the equality $w_{n}\left(x_{n}\right)=\epsilon$ and convergence of the sequence $x_{n}$ to infinity.

Let us also consider the sequence $v_{n}(x)=w_{n}\left(x+x_{n}\right)$ of shifted solutions. Obviously, $v_{n}(0)=\epsilon$. It has a subsequence locally convergent to a monotone solution $v_{0}(x)$ of equation

$$
v^{\prime \prime}+F_{+}(v)=0
$$

defined on the whole axis and such that $v_{0}(+\infty)=0, v_{0}(-\infty)>\epsilon$. Here

$$
F_{+}(v)=\lim _{x \rightarrow \infty} F_{\tau_{0}}(v, x) .
$$

This limit exists by virtue of the condition on the derivative in (2.10).

Set $v_{-}=v(-\infty)$. Then $F_{+}\left(v_{-}\right)=0$. Multiplying equation (2.13) by $v^{\prime}$ and integrating, we obtain 


$$
\int_{0}^{v_{-}} F_{+}(u) d u=0
$$

Thus, the assumption that $x_{\tau}$ is not uniformly bounded leads to the conclusion that there exists a zero $v_{-}$of the function $F_{+}$such that the previous equality holds.

Condition 2.2. For any $v>0$, if $F_{+}(v)=0$, then $\int_{0}^{v} F_{+}(u) d u \neq 0$.

If this condition is satisfied, then the values $x_{\tau}$ are uniformly bounded. Hence the norm $\left\|w_{\tau}\right\|_{\mu}$ is also uniformly bounded.

\subsection{Model problem}

Consider the function $F(w, x)$, which satisfies conditions (2.2)-(2.4), and the limit function

$$
F_{+}(w)=\lim _{x \rightarrow+\infty} F(w, x) .
$$

We suppose that it satisfies the following conditions:

$$
F_{+}^{\prime}(0)<0, \quad F_{+}(w)<0 \text { for } w>w_{+}
$$

with some $w_{+}>0$. Moreover there exists $w_{0} \in\left(0, w_{+}\right)$such that $F_{+}\left(w_{0}\right) \neq 0$,

$$
\int_{0}^{w_{0}} F_{+}(u) d u=0, \quad \int_{0}^{w} F_{+}(u) d u \neq 0 \quad \forall w \in\left(0, w_{+}\right), w \neq w_{0} .
$$

Then problem

$$
w^{\prime \prime}+F_{+}(w)=0, \quad w^{\prime}(0)=0, \quad w(+\infty)=0
$$

has a unique positive solution $w_{0}(x)$, and $w_{0}(0)=w_{0}$ is its maximal value.

We will find the index of this solution, that is the value of the degree with respect to small sphere around this solution. It is given by the following expression:

$$
\operatorname{ind}\left(w_{0}\right)=(-1)^{\nu}
$$

where $\nu$ is the number of positive eigenvalues (with their multiplicities) of the linearized operator

$$
L u=u^{\prime \prime}+F_{+}^{\prime}\left(w_{0}(x)\right) u
$$

acting on functions $C^{2}\left(\mathbb{R}_{+}\right)$.

Lemma 2.5. The eigenvalue problem

$$
u^{\prime \prime}+F_{+}^{\prime}\left(w_{0}(x)\right) u=\lambda u, \quad u^{\prime}(0)=0, \quad u(\infty)=0
$$

does not have a zero eigenvalue. 
Proof. Let us note that the essential spectrum of this problem lies in the left-half plane since $F_{+}^{\prime}(0)<0$. Suppose that the assertion of the lemma does not hold and problem $(2.16)$ has a nontrivial solution $u_{0}(x)$ for $\lambda=0$. Then this solution cannot be positive for all $x$. Indeed, if $u_{0}(x)>0$ for $0 \leq x<\infty$, then the function $v_{0}(x)=u_{0}(|x|)$ defined on the whole axis is a positive solution of the equation

$$
v^{\prime \prime}+F_{+}^{\prime}\left(w_{1}(x)\right) v=0, \quad x \in \mathbb{R},
$$

where the function $w_{1}(x)$ is an extension on the whole axis of the function $w_{0}(x)$ by symmetry. Since the function $v_{0}(x)$ is positive, then $\lambda=0$ is the principal eigenvalue of the operator

$$
L v=v^{\prime \prime}+F_{+}^{\prime}\left(w_{1}(x)\right) v
$$

and this eigenvalue is simple [10]. On the other hand, $v_{1}(x)=w_{1}^{\prime}(x)$ is an eigenfunction of this operator corresponding to the zero eigenvalue, and

$$
v_{1}(x)=-w_{0}^{\prime}(-x)>0, \quad-\infty<x<0, \quad v_{1}(x)=w_{0}^{\prime}(x)<0, \quad 0<x<\infty .
$$

Hence this eigenfunction is not positive, and it is different from the eigenfunction $v_{0}(x)$. We obtain a contradiction with simplicity of the principal eigenvalue.

Thus, the function $u_{0}(x)$ has variable sign. Since it is determined up to a factor, we can assume that $u_{0}(0)<0$. Then it has positive values for some $x>0$ and it decays at infinity since $F_{+}^{\prime}(0)<0$.

Next, the function $u_{1}(x)=-w_{0}^{\prime}(x)$ is a solution of the problem

$$
u^{\prime \prime}+F_{+}^{\prime}\left(w_{0}(x)\right) u=0, \quad u(0)=0, \quad u(\infty)=0,
$$

which differs from problem (2.16) considered for $\lambda=0$ by the boundary condition. This function is positive for all $x>0$. We will use this function to prove that the solution $u_{0}(x)$ cannot exist. Set $\omega(x)=t u_{1}(x)-u_{0}(x)$, where $t$ is a positive number. This function satisfies the equation

$$
\omega^{\prime \prime}+F_{+}^{\prime}\left(w_{0}(x)\right) \omega=0 .
$$

Let $x_{0}$ be such that

$$
F_{+}^{\prime}\left(w_{0}(x)\right)<0, \quad x_{0} \leq x<\infty .
$$

Since $u_{1}(x)$ is a positive function, we can choose $t$ for which $\omega\left(x_{0}\right)>0$. We can verify that

$$
\omega(x)>0, \quad x_{0} \leq x<\infty .
$$

Indeed, if $\omega\left(x_{1}\right)<0$ for some $x_{1}>x_{0}$, then this function has a negative minimum since it converges to 0 at infinity. We obtain a contradiction in signs in equation (2.17) at the point of minimum. If $\omega\left(x_{1}\right)=0$, then we get a contradiction with the maximum principle. Indeed, since $\omega(x) \geq 0$ for $x \geq x_{0}$, then this function is either everywhere positive or identically zero for such $x$. The latter contradicts the assumption that $\omega\left(x_{0}\right)>0$. 
Let us recall that $u_{0}(0)<0$. Therefore $\omega(0)>0$ for any $t>0$. Moreover, (2.18) holds for $t$ large enough. Hence for $t$ sufficiently large, the function $\omega(x)$ is positive for all $x \geq 0$. If $t=0$, then it has negative values since $u_{0}(x)$ has positive values. Let $t_{0}$ be the infimum of all $t$ for which $\omega(x)$ is positive for all $x \geq 0$. Then there exists a value $x_{2} \in\left[0, x_{0}\right]$ for which $\omega\left(x_{2}\right)=0$. Indeed, if $\omega(x)$ is positive in this interval, then $t$ can be decreased in such a way that it remains positive there. Since $\omega\left(x_{0}\right)>0$, then (2.18) holds, and $\omega(x)$ is positive for all $x$. This contradicts the definition of $t_{0}$.

Thus, $\omega(x) \geq 0$ for all $x \geq 0$ and $\omega\left(x_{2}\right)=0$. But this is not possible by virtue of the maximum principle. Hence problem (2.16) cannot have nontrivial solution for $\lambda=0$.

Remark 2.6. The principal eigenvalue $\lambda_{0}$ of problem (2.16) is positive. Indeed, if it is non-positive, then, since the corresponding eigenfunction is positive, we will obtain a contradiction with the fact that the function $-w_{0}^{\prime}(x)$ is a positive (for $x>0$ ) solution of the equation $L u=0$ (cf. the proof of the lemma).

We proved in Lemma 2.5 that this problem does not have a zero eigenvalue. We can verify that any real $\lambda \in\left(0, \lambda_{0}\right)$ is not an eigenvalue of this problem. Indeed, suppose that there is an eigenvalue $\lambda^{*} \in\left(0, \lambda_{0}\right)$. The corresponding eigenfunction $u^{*}(x)$ cannot be positive because only the principal eigenvalue has a positive eigenfunction [10]. As in the proof of the lemma, we introduce the function $\omega=t u_{1}-u^{*}$. It satisfies the equation

$$
\omega^{\prime \prime}+F_{+}^{\prime}\left(w_{0}(x)\right) \omega+\phi(x)=\lambda^{*} \omega,
$$

where $\phi(x)=\lambda^{*} t u_{1}$. As above, we choose $t>0$ in such a way that $\omega(x) \geq 0$ for all $x$ and $\omega\left(x_{2}\right)=0$ for some $x_{2}>0$. Since $\phi(x)>0$ for all $x>0$, then we obtain a contradiction in signs in the last equation at $x=x_{2}$.

\subsection{Existence theorem}

We can now prove the main theorem of this section.

Theorem 2.7. If the function $F(w, x)$ satisfies conditions (2.2)-(2.4) and the function $F_{+}(w)$ satisfies conditions (2.14), (2.15), then problem

$$
\begin{gathered}
w^{\prime \prime}+F(w, x)=0, \\
w^{\prime}(0)=0
\end{gathered}
$$

on the half-axis $x>0$ has a positive monotonically decreasing solution vanishing at infinity. It belongs to the weighted Hölder space $E_{1}$.

Proof. We will consider the homotopy

$$
F_{\tau}(w, x)=\tau F(w, x)+(1-\tau) F_{+}(w) .
$$


Let us recall Condition 2.2, which follows from (2.14), (2.15), and assumption that solutions are uniformly bounded are used for a priori estimates of monotone solutions. The latter can follow from the maximum principle or from the estimates. Conditions (2.14) and (2.15) provide existence of solutions for the model problem; conditions (2.10) and (2.11), which follow from (2.2)-(2.4) and the definition of the function $F_{\tau}(w, x)$, provide separation of monotone solutions.

For $\tau=0$ this equation has a unique strictly decreasing solution $w_{0}(x)$. Let us find its index, that is the degree with respect to a small ball which contains only this solution and no other solutions. Such small ball exists because the operator linearized about this solution is invertible (Lemma 2.5). By virtue of Lemma 2.5 and Remark 2.6

$$
\text { ind }\left(w_{0}\right)=(-1)^{\nu}=-1,
$$

where $\nu$ is the number of positive eigenvalues of the operator linearized about this solution together with their multiplicities [11].

Let us recall that the operator $A_{\tau}: E_{1} \times[0,1] \rightarrow E_{2}$,

$$
A_{\tau}(w)=w^{\prime \prime}+F_{\tau}(w, x)
$$

is proper on closed bounded sets. This means that for any compact set $G \subset E_{2}$ and any closed bounded set $M \subset E_{1} \times[0,1]$, the set $A_{\tau}^{-1}(G) \cap M$ is compact. Therefore the set of solutions of equation (2.8) for all $\tau \in[0,1]$ is compact. By virtue of a priori estimates of monotone solutions, there is a bounded ball $B \subset E_{1}$ which contains all such solutions. Next, because of the separation of monotone and non-monotone solutions, we can construct a domain $D \subset B$ such that it contains all monotone solutions, and it does not contain non-monotone solutions.

Next, monotone solutions of problem (2.8) cannot approach the trivial solution $w \equiv$ 0 in the norm of the function space. Indeed, if $w(0)$ is sufficiently small, then $w^{\prime \prime}(0)=$ $-F_{\tau}(w(0), 0)>0$ and the solution is not monotone since it grows for small $x$ and vanishes at infinity.

Thus, the domain $D$ can be constructed in such a way that it does not contain the trivial solution either. Therefore by virtue of homotopy invariance of the degree $\gamma\left(A_{\tau}, D\right)$ we get

$$
\gamma\left(A_{1}, D\right)=\gamma\left(A_{0}, D\right)=-1 .
$$

Hence equation (2.8) has a positive decaying solution from the space $E_{1}$ for $\tau=1$. The theorem is proved.

Remark 2.8. If we consider equation (2.19) on the whole axis with a function $F(w, x)$ even with respect to $x$ for each $w$, then we can use the result of Theorem 2.7 and extend the solution from the half-axis to the whole axis by symmetry.

Let us also comment on a possible generalization of these results to the multidimensional case. The Leray-Schauder method is based on the topological degree and a priori estimates of solutions. We use the degree construction for Fredholm and proper operators with the 
zero index. It can be applied if the essential spectrum of the linearized operator lies in the left-half plane of the complex plane [11]. This condition is satisfied for the problem under consideration. Hence the degree theory is applicable in the multidimensional case. A priori estimates is the limiting part of the application of the Leray-Schauder method. Some preliminary considerations show that they can be obtained in the radially symmetric case.

It should be also noted that autonomous problems in the whole space possess a zero eigenvalue related to the invariance of solutions with respect to translation. Therefore the index of solution is not defined. Moreover the solution is not isolated but there is a whole family of solutions obtained from each other by translation. In the case of travelling wave solutions, a special technique, functionalization of the parameter was developed in order to deal with this question [10]. In general, it is possible to remove invariance with respect to translation in space considering the subspace orthogonal to the eigenfunction corresponding to the zero eigenvalue.

\subsection{Examples}

Example 2.9. Consider the function

$$
F(w, x)=a w^{2}(1-w)-\sigma(x) w,
$$

where $a>0$ is a constant, $\sigma(x)$ is a positive bounded increasing function, $\sigma_{+}=\sigma(+\infty)$. If problem $(2.8),(2.9)$ has a positive solution, then the estimate $\sup _{x} w(x) \leq 1$ holds. Indeed, otherwise at the point of maximum we obtain a contradiction in signs in the equation.

Let $a>4 \sigma_{+}$. Then equation $a w(1-w)=\sigma_{+}$has two positive solutions. Denote the maximal of them by $v_{-}$. If

$$
v_{-}\left(1-\frac{3}{4} v_{-}\right)>\frac{3 \sigma_{+}}{2 a}
$$

then $\int_{0}^{v_{-}} F_{+}(u) d u>0$, and Condition 2.2 is satisfied. In this case we can apply the result on the existence of solutions. If the integral is negative, the solution may not exist since condition (2.15) is not satisfied.

Example 2.10. Consider, next, the function

$$
F(w, x)=a w^{2}-\sigma(x) w .
$$

Condition 2.2 is obviously satisfied in this case. However we need to estimate the maximum of the solution. It does not follow in this case from the maximum principle since the function $F(w, x)$ is not negative for large $w$. We multiply the equation

$$
w^{\prime \prime}+a w^{2}-\sigma(x) w=0
$$

by $w^{\prime}$ and integrate from 0 to $\infty$. Taking into account that $w^{\prime}(0)=0$ and $w^{\prime}(x)<0$ for all $x>0$, we get 


$$
\frac{a}{3} w^{3}(0)=-\int_{0}^{\infty} \sigma(x) w w^{\prime} d x<\frac{1}{2} \sigma_{+} w^{2}(0)
$$

Hence $\sup _{x} w(x)=w(0)<3 \sigma_{+} / 2 a$. Thus we can obtain a priori estimates and prove existence of solutions by the Leray-Schauder method.

Remark about upper and lower functions. Let us consider as example the function $F$ given by equality (1.4). The condition (2.2) implies that $\sigma^{\prime}(x)>0$. Set

$$
F_{-}(w)=w^{2}(1-w)-\sigma(0) w, \quad F_{+}(w)=w^{2}(1-w)-\sigma(+\infty) w .
$$

Suppose that problems

$$
w^{\prime \prime}+F_{ \pm}(w)=0, \quad w^{\prime}(0)=0, \quad w(+\infty)=0
$$

have solutions and denote them by $w_{-}(x)$ and $w_{+}(x)$, respectively. Their existence depends on the values of $\sigma(0)$ and $\sigma(+\infty)$, and it can be easily verified. Then $w_{-}(x)$ is an upper function and $w_{+}(x)$ is a lower function. If $w_{+}(x) \leq w_{-}(x)$ for all $x \geq 0$, then we can use the method of upper and lower functions to prove the existence of solutions of the problem under consideration. However, this inequality does not hold, and this method is not applicable. On the other hand, the function $w_{-}(x)$ is positive, and it can be considered as an upper function. Then the corresponding solution will decay in time and uniformly converge to the trivial solution $w=0$. Hence it cannot be used to prove the existence of a pulse solution.

\section{Solutions on the half-axis without monotonicity con- dition}

In the previous section we proved existence of monotone solutions on the half-axis using separation of monotone and non-monotone solutions and a priori estimates of monotone solutions. In this section we will study the case without separation of monotone and nonmonotone solutions. In this case we need to obtain a priori estimates of solutions which may not be monotone. We consider problem (2.8), (2.9) on the half-axis assuming that condition (2.11) is satisfied. We do not assume here condition (2.10).

We consider the equation

$$
w^{\prime \prime}+F_{\tau}(w, x)=0
$$

on the half-axis $x>0$ with the boundary condition

$$
w^{\prime}(0)=0 .
$$

We will look for solutions decaying at infinity. Here

$$
F_{\tau}(w, x)=\tau F(w, x)+(1-\tau) F_{+}(w)
$$

where 


$$
F_{+}(w)=\lim _{x \rightarrow \infty} F(w, x) .
$$

The function $F_{\tau}(w, x)$ depends on the parameter $\tau \in[0,1]$. We suppose that it is infinitely differentiable with respect to the variables $w, x, \tau$ and

$$
F_{\tau}(0, x)=0, \quad F_{\tau}(w, x)<0, \quad \forall w>w_{+}, \quad x \geq 0, \quad \tau \in[0,1] .
$$

We will also assume that

$$
F_{+}(0)=0, \quad F_{+}^{\prime}(0)<0 \text { and } \int_{0}^{w} F_{+}(u) d u<0, \quad \forall w \in\left(0, w_{+}\right) .
$$

Proceeding as in Section 2.3, we estimate the values $x_{\tau}$. If they are not uniformly bounded, then the equation

$$
w^{\prime \prime}+F_{+}(w)=0
$$

has a bounded solution $w_{0}(x)$ on the whole axis such that $w_{0}(+\infty)=0$. Then $0 \leq w_{0}(x) \leq$ $w_{0}$ for all $x \in \mathbb{R}$. Moreover either $w_{0}(x)$ is a monotonically decreasing solution with some limit $w_{0}$ at $-\infty$ or it is a pulse solution vanishing at $\pm \infty$ and some maximal value $w_{0}$. In

both cases, $\int_{0}^{w_{0}} F_{+}(u) d u=0$. We obtain a contradiction with inequality (3.4). Thus, we can formulate the following theorem.

Theorem 3.1. If conditions (3.3) and (3.4) are satisfied, then solutions of problem (3.1), (3.2) are uniformly bounded in the norm $C_{\mu}^{2+\alpha}\left(\mathbb{R}_{+}\right)$.

If $\tau=0$, then equation (3.1) coincides with equation (3.5). It has only the trivial solution. The value of the degree with respect to any bounded set in the space $E_{1}$ equals 1 . If $F(0, x) \equiv 0$ for all $x$, then problem (3.1), (3.2) also has the trivial solution. If a simple real eigenvalue of the problem linearized about the trivial solution crosses the origin, then this solution becomes unstable and nontrivial solutions bifurcate from it. This is not only a local bifurcation. There is a continuous branch of solutions starting from the trivial solution.

\section{Existence of pulses in the case of global consumption}

We consider the equation

$$
w^{\prime \prime}+F(w, x, I(w))=0
$$

on the whole axis. It describes evolution of biological species with global consumption of resources [3], [12], [14]. Here

$$
F(w, x, I)=a w^{2}(1-I(w))-\sigma(x) w, \quad I(w)=\int_{-\infty}^{\infty} w(x) d x .
$$

We look for a positive solution decaying at infinity. Set 


$$
c=1-I(w)
$$

and $w=u /(a c)$. Then we get the equation

$$
u^{\prime \prime}+u^{2}-\sigma(x) u=0
$$

which we studied in Section 2.6. If $\sigma(x)$ is a positive even function bounded and increasing for $x>0$, then it has a positive decaying at infinity solution (Example 2.10). Then from $(4.2)$

$$
c^{2}-c+\frac{1}{a} I(u)=0
$$

This equation has two real solutions if $I(u)<a / 4$, one solution in the case of equality, and no solutions if the inequality is opposite. Hence equation (4.1) has pulse solutions if the reproduction rate coefficient $a$ is sufficiently large.

Example 4.1. In the case when $\sigma(x)=\sigma_{0}>0$ is a constant, assuming $u^{\prime}(0)=0$ we can find the analytic solution:

$$
u(x)=\frac{3 \sigma_{0}}{2 \cosh ^{2}\left(\frac{\sqrt{\sigma_{0}}}{2} x\right)}
$$

and the value of the integral $I(u)=6 \sqrt{\sigma_{0}}$. Therefore, in the case of $a>24 \sqrt{\sigma_{0}}$ we have the two pulse solutions given by the formula

$$
w_{1,2}(x)=\frac{3 \sigma_{0}}{2 a c_{1,2} \cosh ^{2}\left(\frac{\sqrt{\sigma_{0}}}{2} x\right)}, \quad c_{1,2}=\frac{1 \pm \sqrt{1-\frac{24}{a} \sqrt{\sigma_{0}}}}{2} .
$$

When $a=24 \sqrt{\sigma_{0}}$, there is a single pulse

$$
w(x)=\frac{3 \sigma_{0}}{\operatorname{acosh}^{2}\left(\frac{\sqrt{\sigma_{0}}}{2} x\right)} .
$$

Finally, for $0<a<24 \sqrt{\sigma_{0}}$, we have no real valued pulse solutions.

\section{References}

[1] A. Ambrosetti, A. Malchiodi. Nonlinear analysis and semilinear elliptic problems. Cambridge Univ. Press, Cambridge, 2007.

[2] H. Berestycki, P.L. Lions, L.A. Peletier. An ODE approach to the existence of positive solutions for semilinear problems in $\mathbb{R}^{N}$. Indiana Univ. Math. J., 30 (1981), no.1, 141157. 
[3] N. Bessonov, N. Reinberg, V. Volpert. Mathematics of Darwin's diagram. Math. Model. Nat. Phenom., 9 (2014), no. 3, 5-25.

[4] L.A. Caffarelli, B. Gidas, J. Spruck. Asymptotic symmetry and local behavior of semilinear elliptic equations with critical Sobolev growth. Comm. Pure Appl. Math., 42 (1989), no. 3, 271-297.

[5] W.X. Chen, C. Li. Classification of solutions of some nonlinear elliptic equations. Duke Math. J., 63 (1991), no. 3, 615-622.

[6] W.X. Chen, C. Li. Qualitative properties of solutions to some nonlinear elliptic equations in $R^{2}$. Duke Math. J., 71 (1993), no. 2, 427-439.

[7] B. Gidas, J. Spruck. Global and local behavior of positive solutions of nonlinear elliptic equations. Comm. Pure Appl. Math., 34 (1981), no. 4, 525-598.

[8] B. Gidas, J. Spruck. A priori bounds for positive solutions of nonlinear elliptic equations. Comm. Partial Differential Equations, 6 (1981), no. 8, 883-901.

[9] I. Kuzin, S. Pohozaev. Entire solutions of semilinear elliptic equations. Birkhäuser, Basel, 1997.

[10] A.I. Volpert, V. Volpert, V.A. Volpert. Traveling wave solutions of parabolic systems. Translation of Mathematical Monographs, Vol. 140, AMS, Providence, 1994.

[11] V. Volpert. Elliptic partial differential equations. Volume 1. Fredholm theory of elliptic problems in unbounded domains. Birkhäuser, 2011.

[12] V. Volpert. Elliptic partial differential equations. Volume 2. Reaction-diffusion equations. Birkhäuser, 2014.

[13] V. Volpert. Pulses and waves for a bistable nonlocal reaction-diffusion equation. Applied Mathematics Letters, 44 (2015), 21-25.

[14] V. Volpert, N. Reinberg, M. Benmir, S. Boujena. On pulse solutions of a reactiondiffusion system in population dynamics Nonlinear Analysis 120 (2015), 76-85.

[15] V. Volpert, V. Vougalter. Existence of stationary pulses for nonlocal reaction-diffusion equations, Doc. Math., 19 (2014), 1141-1153.

[16] V. Vougalter, V. Volpert. On the existence of stationary solutions for some integrodifferential equations. Doc. Math., 16 (2011), 561-580. 